\title{
Cobertura vacinal e fatores associados em puérperas de município paulista
}

\author{
Immunization coverage and associated factors of women \\ who have recently given birth in a city in São Paulo state
}

Bárbara Cristina Casemiro da Rocha ${ }^{1}$
Ana Paula Pinho Carvalheira ${ }^{1}$
Anna Paula Ferrari ${ }^{1}$
Vera Lúcia Pamplona Tonete $^{1}$
Marli Teresinha Cassamassimo Duarte $^{1}$
Cristina Maria Garcia de Lima Parada

\begin{abstract}
The importance of immunization among actions recommended in the prenatal period raises the possibility of protecting both the mother and the fetus against certain harmful diseases. The scope of this study was to assess the immunization coverage and associated factors of mothers whose deliveries occurred in the first half of 2012 in Botucatu, state of São Paulo. It is a cross-sectional study and data collection was performed in the two municipal maternity hospitals. Of the total births during the study period, 1318 mothers (90.3\%) were included The data for the characterization of participants and their vaccination status (diphtheria, tetanus and hepatitis B) were obtained from the prenatal card, hospital records or interviews. For analysis of associated factors, the Chi-square test was used, with a 95\% confidence interval and critical $p$ of $<0.05$. Most of the study participants were 20 years old or more (84.4\%), had seven years of schooling (69\%), were monitored in prenatal care (99.2\%) with seven or more appointments (88.9\%). Miscarriage and infant death of $17.7 \%$ and $2.4 \%$ was recorded, respectively. Vaccination coverage was $68.4 \%$. There was no association between sociodemographic and obstetric variables relating to prenatal care and vaccination of pregnant women.
\end{abstract}

Key words Pregnant women, Immunization, Prenatal care, Immunization coverage.
Resumo A importância da imunização entre as ações preconizadas no pré-natal decorre da possibilidade de proteger a mãe e o concepto contra determinadas doenças reconhecidamente prejudiciais a ambos. O objetivo do presente estudo foi avaliar a cobertura vacinal de puérperas cujos partos ocorreram no primeiro semestre de 2012 em Botucatu/SP e fatores associados. Trata-se de estudo transversal. A coleta de dados foi realizada nas duas maternidades do municipio. Foram incluídas 1318 puérperas, 90,3\% do total de partos ocorridos no período. Os dados para a caracterização das participantes e sobre a situação vacinal (dupla adulto e contra hepatite B) foram obtidos do cartão de pré-natal, prontuário hospitalar ou por entrevista com as mesmas. Para análise dos fatores associados utilizou-se teste Qui-quadrado, considerando-se intervalo de confiança de $95 \%$ e p crítico $<0,05$. A maior parte das participantes tinha 20 anos ou mais (84,4\%), até sete anos de aprovação escolar (69,0\%), foi acompanhada no pré-natal $(99,2 \%)$ realizando sete ou mais consultas (88,9\%). Tinham história de aborto e de óbito infantil 17,7\% e 2,4\% das puérperas, respectivamente. A cobertura vacinal foi de $68,4 \%$. Não houve associação entre variáveis sociodemográficas, obstétricas, relativas ao pré-natal e vacinação da gestante.

Palavras-chave Gestantes, Imunização, Cuidado pré-natal,Cobertura vacinal

\footnotetext{
Montenegro s/n, UNESP. 18618-687 Botucatu SP Brasil.babi.casemiro@ hotmail.com

Enfermagem, Faculdade de Medicina de Botucatu, Universidade Estadual Paulista. Av. Prof. Mário
} 


\section{Introdução}

A vacinação na gestação protege não apenas a mãe, mas também o recém-nascido, pela passagem de anticorpos, tanto pela via transplacentária, quanto pela amamentação. Objetiva-se proteger a criança de patógenos que causam infecções durante os primeiros meses de vida, período de sua maior vulnerabilidade ${ }^{1}$. Assim, a vacinação é importante intervenção a ser desenvolvida rotineiramente durante o acompanhamento prénatal.

Não há evidências de que a administração de vacinas compostas por toxoides, como o tetânico e o diftérico ${ }^{2-4}$ ou por antígeno recombinante de superfície do vírus da hepatite $\mathrm{B}^{2,5}$ acarretem qualquer risco para o feto quando administradas em gestantes.

O calendário vacinal da gestante vem sendo ampliado no país. Atualmente, são indicadas a vacina adsorvida contra difteria e tétano $(\mathrm{dT})$ ou a vacina antitetânica apenas, a vacina recombinante contra hepatite B e contra influenza, esta última durante os meses do outono e inverno ${ }^{2}$. Em 2014, também passou a ser indicada a vacina acelular contra difteria, tétano e coqueluche (dTpa), especialmente para estímulo à produção de anticorpos maternos contra essa última ${ }^{2-5}$.

Pela incipiência da utilização da dTpa e a sazonalidade da vacinação contra influenza, este estudo terá como foco a cobertura vacinal da dT e contra a hepatite $B$ na gestação.

Com a implementação da política de eliminação do tétano neonatal como problema de saúde pública mundial, sua incidência diminuiu drasticamente, principalmente nas Américas. Segundo a Organização Mundial de Saúde, a meta de alcançar a taxa de incidência de menos de um caso por mil nascidos vivos de forma homogênea nos países, ainda não foi alcançada em situações de menor desenvolvimento econômico e social ${ }^{6}$.

No Brasil, o tétano integra o elenco de agravos de notificação compulsória desde os anos de 1980 e sua incidência diminuiu de 291 casos em 1990 para três em 2013: um na região sudeste e dois na região norte, falha grave considerando tratar-se de doença imunoprevenível ${ }^{7}$. Estratégia prioritária para a eliminação do tétano neonatal, a vacinação de gestantes ainda é falha no Brasil: estudo nacional apontou que em 31\% dos nascimentos do país, a mãe não havia tomado dose alguma da vacina ${ }^{8}$.

Da mesma forma que o tétano, a hepatite B é doença grave no período neonatal ${ }^{9}$. A transmissão vertical da hepatite B é responsável por
$35 \%$ a $40 \%$ dos novos casos da doença, pois é por meio dela que o vírus é mantido na população, fato que justifica a importância da vacinação de gestantes $^{10}$.

A vacina contra a hepatite B é altamente imunogênica e, apesar do uso seguro na gravidez, ainda é um desafio para o Brasil tornar sua cobertura universal: há aproximadamente dez anos mantém-se próxima a $90 \%{ }^{11}$. Está indicada para gestantes com sorologia negativa para essa doença e que perderam a oportunidade de recebê-la na rotina dos serviços de saúde. Espera-se que essa estratégia contribua para a redução do potencial de transmissão vertical dessa doença e da tendência a sua cronificação, situação frequente quando a doença ocorre em idade precoce ${ }^{10,11}$.

Reconhecendo a importância da vacinação e a possível dificuldade de incorporar essa tecnologia na rotina pré-natal, bem como o desconhecimento sobre pesquisas nacionais nessa temática, propõe-se a realização do presente estudo, que tem por objetivo avaliar a cobertura vacinal de puérperas cujos partos ocorreram no primeiro semestre de 2012 em Botucatu/SP e fatores associados.

\section{Material e método}

Trata-se de estudo transversal. O cenário da pesquisa é um município com aproximadamente 127.000 habitantes $^{12}$, localizado na região centrosul do Estado de São Paulo e que conta com duas maternidades, uma pública e outra privada.

A maternidade pública atende em média 180 parturientes por mês e possui 29 leitos, sendo nove cirúrgicos e 20 clínicos. É referência nas áreas obstétrica e neonatal, em especial para os 68 municípios que compõem o Departamento Regional de Saúde VI - Bauru. A maternidade privada possui 16 leitos, incluindo quartos privativos e semiprivativos e, em média, ocorrem 70 partos por mês.

A coleta de dados foi realizada no período de seis meses, a partir de $1^{\circ}$ de janeiro de 2012 , por equipe de entrevistadores remunerados e devidamente capacitados. Foram critérios de inclusão: ter parto em uma das duas maternidades existentes em Botucatu e concordar em participar do estudo. Dos 1416 partos ocorridos no referido período, foram incluídos 1318 (93,1\%), havendo 91 perdas e sete recusas.

A variável desfecho estudada foi vacinação completa de dT e contra hepatite B ( $\operatorname{sim} /$ não), obtida a partir de entrevista com as puérperas, 
realizada no mínimo 12 horas após o parto e antes da alta hospitalar. As variáveis independentes foram: sociodemográficas e relativas a antecedentes obstétricos e parto atual (obtidas dos prontuários e cartão de pré-natal) e relacionadas ao pré-natal (obtidas por entrevista com as puérperas), a seguir apresentadas.

Sociodemografia: faixa etária em anos (até 19, 20 ou mais); sem companheiro (sim, não); anos de aprovação escolar (até sete, oito ou mais); cor (não branca, branca) e procedência (outros municípios, Botucatu).

Antecedentes obstétricos e parto atual: primigestação ( $\operatorname{sim}$, não); abortos anteriores (sim, não) e filhos mortos anteriores (sim, não); parto prematuro (sim, não)

Pré-natal: acompanhamento pré-natal (não fez, fez); número de consultas pré-natais (até seis, sete ou mais); local do pré-natal (serviço público, serviço privado ou conveniado); prénatal deficiente e ausência de atividade educativa em grupo (sim, não). Para compor a variável pré-natal deficiente optou-se por verificar se a puérpera havia recebido orientações pré-natais sobre aspectos relevantes, como: alimentação na gestação, sinais de alerta no termo e aleitamento materno (sim, não).

A consistência do banco de dados foi avaliada a partir de questões associadas, sendo corrigidas todas as falhas identificadas. A análise de dados foi realizada empregando-se o software EpiInfo 7.1.3.3. Apresentam-se os dados de forma descritiva e, na busca por associações, utilizou-se o teste Qui-quadrado, considerando-se intervalo de confiança de 95\% e p < 0,05 como nível crítico.

Este estudo foi aprovado por Comitê de Ética em Pesquisa e seguiu todas as normas preconizadas para pesquisa envolvendo seres humanos.

\section{Resultados}

As características estudadas das participantes do estudo constam da Tabela 1 .

A maioria das mulheres tinha 20 anos ou mais $(84,4 \%)$, possuía companheiro $(85,6 \%)$, era da raça/cor branca $(78,5 \%)$, com até sete anos de aprovação escolar $(69,0 \%)$, havia realizado prénatal no serviço público $(69,1 \%)$ e era procedente do município foco desta pesquisa $(60,2 \%)$. Considerando os dados obstétricos, a maioria (62,7\%) já tinha passado por gestações anteriores, não tinha história de óbito infantil $(97,6 \%)$ e nem de aborto $(82,3 \%)$. A cobertura pré-natal foi de $99,2 \%$, sendo que $88,9 \%$ das mulheres tiveram sete ou mais consultas e $85,6 \%$ delas tiveram parto a termo (Tabela 1 ).

A cobertura vacinal considerando-se o esquema completo da dT e contra a hepatite B foi $68,4 \%$. A prevalência de pré-natal não deficiente foi de $63,2 \%$ das mulheres e, neste caso, elas tinham recebido orientações sobre sinais de alerta no termo, alimentação na gestação e aleitamento materno. Mencionaram não ter participado de atividade educativa em grupo 77,4\% das puérperas (Tabela 1 ).

Quando se buscou associação entre ter esquema vacinal completo no puerpério (dT e contra hepatite B) e variáveis sociodemográficas, obstétricas e relativas ao pré-natal, pôde-se observar que não houve qualquer associação (Tabela 1).

\section{Discussão}

O presente estudo analisou a situação vacinal de puérperas cujos partos ocorreram no primeiro semestre de 2012, em município do interior paulista, buscando identificar fatores associados à vacinação. Trata-se do primeiro estudo nacional que avalia a cobertura vacinal de gestantes considerando duas das vacinas atualmente preconizadas: vacina dT e contra hepatite B.

Segundo o Ministério da Saúde (MS) brasileiro, no contexto do cuidado integral à saúde da mulher, a atenção pré-natal deve ser organizada para atender às necessidades da população de gestantes, mediante a utilização dos conhecimentos técnico-científicos existentes e dos meios e recursos disponíveis mais adequados para cada caso. Dessa forma, para a assistência pré-natal efetiva, dentre outras ações, deve-se procurar garantir a vacinação dT e contra hepatite $\mathrm{B}^{13}$.

A opção pela inclusão de ambas as vacinas neste estudo decorreu do fato de estar em pauta, nos estudos sobre avaliação pré-natal, a necessidade de abordagem do conjunto de ações preconizadas e não de ações ou indicadores isolados ${ }^{14}$.

Pesquisa realizada em um dos sete países que não eliminou o tétano materno e neonatal em mais da metade de seus distritos destacou como problema prioritário a baixa cobertura vacinal ${ }^{15}$. Este problema foi, também, apontado com destaque em estudo chinês sobre causas relacionadas à transmissão materno-infantil do vírus da hepatite $\mathrm{B}^{16}$.

Na presente investigação a cobertura das vacinas dT e contra hepatite B foi de 68,4\%. Estudos nacionais, apenas sobre a vacina contra tétano, apontaram valores entre $58,5 \%$ e $76,9 \%{ }^{17}$. 
Tabela 1. Associação entre ter esquema vacinal completo no puerpério (dT e contra hepatite B) e variáveis sociodemográficas, obstétricas e relativas ao pré-natal. Botucatu, 2012,

\begin{tabular}{|c|c|c|c|}
\hline Variáveis & $\begin{array}{c}\text { Total } \\
\text { Vacinada } \\
\mathrm{N}^{\circ}(\%)^{*}\end{array}$ & $\begin{array}{l}\text { Vacinada } \\
\mathrm{N}^{\mathrm{o}}(\%)^{* *}\end{array}$ & p valor \\
\hline Faixa etária em anos & & & 0,7899 \\
\hline Até 19 & $198(15,6)$ & $137(69,2)$ & \\
\hline 20 ou mais & $1069(84,4)$ & $726(67,9)$ & \\
\hline Anos de aprovação escolar & & & 0,5445 \\
\hline Até 7 & $879(69,0)$ & $598(68,0)$ & \\
\hline 8 ou mais & $398(31,0)$ & $272(68,3)$ & \\
\hline Raça/cor & & & 0,5547 \\
\hline Não Branca & $272(21,5)$ & $183(67,3)$ & \\
\hline Branca & $996(78,5)$ & $686(68,9)$ & \\
\hline Sem companheiro & & & 0,9709 \\
\hline Sim & $182(14,4)$ & $124(68,1)$ & \\
\hline Não & $1079(85,6)$ & $735(68,1)$ & \\
\hline Local do pré-natal & & & 0,6644 \\
\hline Serviço público & $794(69,1)$ & $541(68,1)$ & \\
\hline Serviço privado/convênios & $355(30,9)$ & $243(68,4)$ & \\
\hline Procedência & & & 0,8833 \\
\hline Outros municípios & $524(39,8)$ & $357(68,1)$ & \\
\hline Botucatu & $794(60,2)$ & $544(68,5)$ & \\
\hline Primigestação & & & 0,9845 \\
\hline Sim & $470(37,3)$ & $319(67,9)$ & \\
\hline Não & $789(62,7)$ & $538(68,2)$ & \\
\hline Óbito infantil anterior & & & 0,7151 \\
\hline Sim & $30(2,4)$ & $24(80,0)$ & \\
\hline Não & $1234(97,6)$ & $845(68,5)$ & \\
\hline Aborto anterior & & & 0,5997 \\
\hline Sim & $230(17,7)$ & $161(70,0)$ & \\
\hline Não & $1067(82,3)$ & $728(68,2)$ & \\
\hline Consultas pré-natais & & & 0,3258 \\
\hline Não fez & $11(0,8)$ & $6(54,5)$ & \\
\hline $\mathrm{Fez}$ & $1307(99,2)$ & $790(60,4)$ & \\
\hline Número de consultas pré-natais & & & 0,4608 \\
\hline Até 6 & $145(11,1)$ & $66(45,5)$ & \\
\hline 7 ou mais & $1162(88,9)$ & $496(42,7)$ & \\
\hline Ausência de grupo no pré-natal & & & 0,6761 \\
\hline Sim & $1015(77,4)$ & $691(68,1)$ & \\
\hline Não & $297(22,6)$ & $206(69,4)$ & \\
\hline Pré-natal deficiente & & & 0,0657 \\
\hline Sim & $475(36,8)$ & $310(65,3)$ & \\
\hline Não & $817(63,2)$ & $586(71,7)$ & \\
\hline Parto prematuro & & & 0,8878 \\
\hline Sim & $188(14,4)$ & $129(68,6)$ & \\
\hline Não & $1119(85,6)$ & $762(58,3)$ & \\
\hline
\end{tabular}

* Cálculo na coluna. ${ }^{* *}$ Cálculo na linha.

Valores abaixo desses eram esperados, por somarem-se duas vacinas. De toda forma, a cobertura obtida pode ser considerada baixa, pela disponibilidade das vacinas e de recursos físicos, materiais e equipes capacitadas, em todas as unidades de saúde do município estudado. Não foram encontrados na literatura estudos sobre a cobertura da vacina contra hepatite $B$ em gestantes.

Esperava-se, também, relação direta entre vacinação e melhores condições sociodemográ- 
ficas: gestantes não adolescentes, com companheiro, com mais alta escolaridade, brancas e com atendimento pré-natal em serviços privados ou conveniados, associação não confirmada. Da mesma forma, não foram favorecidas as mulheres residentes no município em estudo, mesmo sendo esse o único da região com salas de vacinas informatizadas e controle eletrônico das doses administradas, tanto em campanhas quanto na rotina do serviço, condição que deveria contribuir para redução da perda de oportunidades de vacinação.

A multiparidade e os antecedentes obstétricos desfavoráveis, como abortos e óbitos infantis, não ampliaram a chance de vacinação. Assim, não se confirmou a ideia de que gestantes com história de problemas em outras gestações seriam mais aderentes aos cuidados pré-natais, inclusive à vacinação; tampouco ocorreu vantagem entre multíparas, pela possibilidade de vacinação em gestações prévias. Acrescenta-se que não foi verificado o favorecimento em relação à vacinação das mulheres que tiveram parto a termo, indicando que falhas no pré-natal não ocorrem apenas quando o parto acontece antes do esperado.

Com relação ao cuidado pré-natal, esperava-se associação entre maior cobertura vacinal e casos mais favoráveis: número de consultas igual ou superior a sete, participação em atividade educativa em grupo e classificação do pré-natal como não deficiente. Tal associação, porém, não foi observada. Esse fato corrobora com estudos brasileiros realizados nos últimos dez anos, que têm apontado baixa qualidade dos serviços de pré-natal mesmo em situação de elevado número de consultas ${ }^{18,19}$. Melhorar a qualidade do cuidado à gestante, o que pode ser considerada uma forma de vigilância em saúde, também é desafio para o município onde este estudo foi conduzido.
Destaca-se, porém, duas limitações deste estudo: as puérperas não foram questionadas quanto aos motivos para a não vacinação: se por opção ou desconhecimento e o fato das vacinas incluídas terem sido analisadas em conjunto.

\section{Conclusão}

Este estudo permitiu conhecer a situação vacinal de gestantes, considerando não apenas a vacina $\mathrm{dT}$, mas também contra hepatite $\mathrm{B}$, buscando fatores associados, com potencialidade de contribuir com a formulação de políticas públicas municipais, visando à melhoria do atendimento à saúde materna no município deste estudo e em outros, com condições semelhantes.

Não houve associação entre a baixa cobertura vacinal encontrada e as principais variáveis apontadas como possíveis determinantes do atraso/não realização vacinal: piores condições sociais, vivência de desfechos negativos anteriores e atenção ao pré-natal deficiente. Os resultados obtidos apontam para a falta da integralidade no cuidado pré-natal desenvolvido no município deste estudo, especialmente ao se considerar a quase universal cobertura dessa prática, inclusive com elevada proporção de mulheres com sete ou mais consultas. Por outro lado, a impossibilidade de identificar um grupo com maior chance de não vacinação indica a necessidade de atentar igualmente para o conjunto de gestantes, de forma a viabilizar a ampliação desta importante ação preventiva de saúde.

Sugere-se, por fim, a realização de estudos com outros desenhos metodológicos voltados a compreender, na perspectiva das próprias gestantes e dos profissionais de saúde, razões para a baixa cobertura vacinal.

\section{Colaboradores}

BCC Rocha trabalhou na elaboração do projeto de pesquisa, do artigo e na coleta de dados. APP Carvalheira e AP Ferrari trabalharam na análise dos dados. VLP Tonete e MTC Duarte trabalharam na revisão crítica do artigo. CMGL Parada trabalhou em todas as etapas, desde a concepção até revisão final do artigo. 


\section{Agradecimentos}

À Fundação de Amparo à Pesquisa do Estado de São Paulo (FAPESP) pelo auxílio à pesquisa.

\section{Referências}

1. Louzeiro EM, Queiroz RCCS, Souza IBJ, Carvalho LKCAA, Araújo TME. A importância da vacinação em gestantes: uma revisão sistemática da literatura no período de 2003 a 2012. Rev Interd 2014; 7(1):193-203.

2. Brasil. Ministério da Saúde (MS). Secretaria de Vigilância em Saúde. Departamento de Vigilância das Doenças Transmissíveis. Manual de Normas e Procedimentos para Vacinação. Brasília: MS; 2014.

3. Keller-Stanislawski B, Englundb JA, Kangc G, Mangtani P, Neuzil K, Nohynekf H, Pless R, Lambachh P, Zuber P. Safety of immunization during pregnancy: a review of the evidence of selected inactivated and live attenuated vaccines. Vaccine 2014; 32(52):7057-7064.

4. Centers for Disease Control and Prevention. Updated recommendations for use of Tetanus Toxoide, Reduced Diphtheria Toxoide, and Acellular Pertussis Vaccune (Tdap) in pregnant women - Advisory Committee on Immunization Practices (ACIP), 2012. MMWR 2013; 62( $\mathrm{N}^{\circ} \mathrm{RR}$ 07):131-135.

5. Centers for Disease Control and Prevention. A Comprehensive Immunization Strategy to Eliminate Transmission of Hepatitis B Virus Infection in the United States Recommendations of the Advisory Committee on Immunization Practices (ACIP) Part II: Immunization of Adults. MMWR 2006; 55( $\mathrm{N}^{\circ}$ RR 16):1-40.

6. Roper MH, Vandelaer JH, Gasse FL. Maternal and neonatal tetanus. The Lancet - 12 de setembro de 2007. [online] [acessado 28 ago 2015]. Disponível em: http:// www.who.int/immunization/diseases/Maternal_and_ neonatal_tetanus_Seminar.pdf

7. Brasil. Ministério da Saúde (MS). Portal da Saúde SUS. Tétano neonatal. [acessado $11 \mathrm{dez} 2014$ ]. Disponível em: http://portalsaude.saude.gov.br/index.php/o-ministerio/principal/secretarias/svs/tetano-neonatal

8. Brasil. Ministério da Saúde (MS). Centro Brasileiro de Análise e Planejamento. Pesquisa Nacional de Demografia e Saúde da Criança e da Mulher - PNDS 2006: dimensões do processo reprodutivo e da saúde da criança. Brasília: MS; 2009.

9. Poovorawan $\mathrm{Y}$, Chongsrisawat $\mathrm{V}$, Theamboonlers A, Leroux Roels G, Kuriyakose S, Leyssen M, Jacquet JM. Evidence of protection against clinical and chronic hepatitis B infection 20 years after infant vaccination in a high endemicity region. J Viral Hepat 2011; 18(5):369-375.

10. Machado Filho AC, Sardinha JFJ, Ponte RL, Costa EP, Silva SS, Espinoza FEM. Prevalência de infecção por HIV, HTLV, VHB e de sífilis e clamídia em gestantes numa unidade de saúde terciária na Amazônia ocidental brasileira. Rev Bras Ginecol Obstet 2010; 32(4):176-183.
11. Grangeiro GR, Diógenes MAR, Moura ERF. Atenção pré-natal no Município de Quixadá-CE segundo indicadores de processo do SISPRENATAL. Rev Esc Enferm USP 2008; 42(1):105-111.

12. Instituto Brasileiro de Geografia e Estatística (IBGE). Resultados preliminares do Universo do Censo Demográfico, 2010. [online] [acessado 24 set 2011]. Disponível em: http://www.ibge.gov.br/cidadesat/topwindow.htm?1

13. Brasil. Ministério da Saúde (MS). Secretaria de Atenção à Saúde. Departamento de Atenção Básica. Atenção ao pré-natal de baixo risco. Brasília: Editora do Ministério da Saúde; 2012. (Série A. Normas e Manuais Técnicos. Cadernos de Atenção Básica no 32).

14. Oliveira RLA, Fonseca CRB, Carvalhaes MABR, Parada CMGL. Avaliação da atenção pré-natal na perspectiva dos diferentes modelos na atenção primária. Rev. Latino-Am. Enfermagem 2013; 21(2):546-553.

15. Masuno K, Xaysomphoo D, Phengsavanh A, Douangmala S, Kuroiwa C. Scaling up interventions to eliminate neonatal tetanus: factors associated with the coverage of tetanus toxoid and clean deliveries among women in Vientiane, Lao PDR. Vaccine 2009; 27(32):4284-4288.

16. Kang W, Ding Z, Shen L, Zhao Z, Huang G, Zhang J, Xiong Q, Zhang S, Zhang S, Wang F. Risk factors associated with immunoprophylaxis failure against mother to child transmission of hepatitis B virus and hepatitis $B$ vaccination status in Yunnan province, China. Vaccine 2014; 32(27):3362-3366.

17. Serruya SJ, Lago TG, Cecatti JG. Avaliação preliminar do programa de humanização no pré-natal e nascimento no Brasil. RBGO 2014; 26(7):517-525.

18. Gonçalves CV, César JA, Sassi RAM. Qualidade e equidade na assistência à gestante: um estudo de base populacional no Sul do Brasil. Cad Saude Publica 2009; 25(11):2507-2516.

19. Villas EF, Domingues RMSM, Dias MAB, Gama SGN, Theme-Filha MM, Costa JV, Bastos MH, Leal MC. Assistência pré-natal no Brasil. Cad Saude Publica 2014; 30(Supl1):S85-S100.

Artigo apresentado em 12/05/2015

Aprovado em 15/09/2015

Versão final apresentada em 17/09/2015 